\title{
A NEGATIVE ANSWER TO THE PRIME SEQUENCE QUESTION
}

\author{
RAYMOND C. HEITMANN
}

\begin{abstract}
If $\boldsymbol{P}$ is a complete intersection prime, i.e., a prime ideal generated by $h t(P)$ elements, in a Noetherian domain, can $P$ be generated by a prime sequence, a regular sequence whose initial segments generate prime ideals? The purpose of this article is to present an example showing that this question, the Prime Sequence Question, has a negative answer. The example is a two-dimensional integrally closed domain with a height two complete intersection prime which contains no prime elements.
\end{abstract}

The prime sequence question was first raised publicly by J. Ohm in [Math. Reviews 54 \#5206]. It is discussed in [1] and it is shown to have an affirmative answer for certain types of rings. However, the prevailing belief was that the general case would have a negative answer and here we shall demonstrate precisely that. The example given here is closely based on the construction in [3]. In light of the large number of alterations which are necessary, it seems preferable to make the construction here independent of the earlier construction. Consequently, familiarity with [3] will not be necessary.

Notation. Let $K$ denote a fixed countable field. Let $X_{1}, X_{2},\left\{Y_{i}\right\}_{i=1}^{\infty}$ be indeterminates. The subscript will be deleted when we wish to denote a family of indeterminates, e.g., $Y=\left\{Y_{i}\right\}$. Fix a set $A$ of irreducible polynomials of $K[X, Y]$, choosing precisely one generator for each height one prime of $K[X, Y]$ contained in the ideal $\left(X_{1}, X_{2}\right)$.

Construction. Since $A$ is countable, we may enumerate its elements $f_{1}, f_{2}, \ldots$ Moreover, it is easy to do this in such a way that $f_{k} \in$ $K\left[X_{1}, X_{2}, Y_{1}, \ldots, Y_{k-1}\right]$. Using this, define new elements $Z_{k}=f_{k}^{2} / Y_{k}$. Next we define a family of rings.

For $k>0$, set $R_{k}=\left(K\left(Y-\left\{Y_{k}\right\}, Z_{k}\right)[X]\right)_{\left(f_{k}\right)}$, the localization of a polynomial ring at a height one prime and consequently a discrete valuation ring. We let $v_{k}$ denote the appropriate valuation. Note that our method of indexing the elements of $A$ guarantees that $R_{k}$ is well defined. For $k=0$, set $R_{0}=$ $(K(Y)[X])_{S}$ where $S$ is the multiplicative set consisting of all products of elements in

Received by the editors July 29, 1978 and, in revised form, November 7, 1978.

AMS (MOS) subject classifications (1970). Primary 13A15, 13F99.

Key words and phrases. Noetherian ring, prime sequence, complete intersection prime, valuation. 


$$
S^{\prime}=\left\{\begin{array}{l|l}
Y_{m}^{n+1}+\sum_{i=0}^{n} a_{i} Y_{m}^{i} & \begin{array}{l}
a_{i} \in K\left[X, Y-\left\{Y_{m}\right\}\right] \\
v_{m}\left(a_{n}\right)=0 \\
v_{m}\left(\sum a_{i} Y_{m}^{i}\right)=2 n
\end{array}
\end{array}\right\} \cup\left\{Y_{m}^{3}+f_{m}^{4}\right\}
$$

Finally, set $R=\cap \bigcap_{k=0}^{\infty} R_{k}$. We shall show that $R$ is the desired example.

LEMMA 1. $K[X, Y, Z] \subset R$.

Proof. It suffices to show $K[X, Y, Z] \subset R_{k}$ for each $k$. Obviously, $K[X]$ $\subset R_{k}$; likewise, for $i \neq k, Y_{i} \in R_{k}$ and $Z_{k} \in R_{k}$. Further, since $f_{k} \in R_{k}$ and $Z_{k}$ is a unit of $R_{k}, Y_{k} \in R_{k}$. Thus, $Y \subset R_{k}$ and so, for each $i, f_{i} \in R_{k}$. When $i \neq k, Y_{i}$ is a unit of $R_{k}$ and therefore $Z_{i} \in R_{k}$.

LEMMA 2. For each $k \geqslant 0, R_{k}$ is a localization of $R$ and so a flat algebra over $R$.

Proof. The first half of the statement is immediate from the definition of $R_{k}$ and Lemma 1. Further, localizations are always flat algebras.

LEMMA 3. Every element of $R$ is a nonunit in at most finitely many $R_{k}$.

Proof. Since $K[X, Y] \subset R \subset K(X, Y)$, it suffices to consider elements of $K[X, Y]$. Such an element $g$ involves only finitely many indeterminates and is divisible, in the polynomial ring $K[X, Y]$, by only finitely many $f_{i}$. Hence, there exists an $N$ such that $g \in K\left[Y_{1}, \ldots, Y_{N}, X_{1}, X_{2}\right]$ and $g \notin f_{k} K[X, Y]$ for all $k>N$. Then $g \notin f_{k} K\left[Y-\left\{Y_{k}\right\}, Z_{k}, X\right]$ and hence also $\notin f_{k} K(Y-$ $\left.\left\{Y_{k}\right\}, Z_{k}\right)[X]$; so $g$ is a unit of $R_{k}$ for all $k>N$.

LEMMA 4. For any nonzero prime $P$ of $R$, there exists a $k$ and a prime $Q$ of $R_{k}$ so that $P=Q \cap R$. Clearly then, $P R_{k} \neq R_{k}$.

Proof. First we assert that $P=\cup\left(P_{k i} \cap R\right)$ where $P_{k i}$ is a nonzero prime of $R_{k}$ and the union is taken over all $P_{k i} \cap R \subset P$. The verification is straightforward and we shall not include it here. In fact, precisely this item is shown in [3, p. 671] in the first paragraph of the proof of Lemma 4.

Next we claim that only a single $k$ occurs in the representation $P=\cup\left(P_{k i}\right.$ $\cap R$ ). If the claim is valid, $P$ contains no units of $R_{k}$ and so $P R_{k} \neq R_{k}$. Inasmuch as $R_{k}$ is a localization of $R$ and there is a one-to-one correspondence between primes of $R_{k}$ and primes of $R$ which do not blow up in $R_{k}, P$ must correspond to a prime $Q$ of $R_{k}$, i.e., $P=Q \cap R$. So it suffices to verify the claim, which we shall do by contradiction.

Suppose $P_{m i} \cap R, P_{n i} \cap R \subset P$ where $m \neq n$. There are two cases to consider: either both $m, n$ are nonzero or one of them, say $n$, is zero. In the first case, note that $P_{m i}$ must be the unique nonzero prime of $R_{m}$. Therefore, $Y_{m}=\left(1 / Z_{m}\right) f_{m}^{2} \in P_{m i}$. Thus, $Y_{m}$ (and similarly $Y_{n}$ ) is in $P$. This yields $\left(Y_{m}+Y_{n}\right) \in P$. However, the latter element is a unit in each $R_{k}$ and so in $R$ as well-a contradiction.

Therefore, we may assume $P=\left(P_{m} \cap R\right) \cup\left(\cup\left(P_{0 i} \cap R\right)\right)$. Consider the 
subring $T=K\left[X, Y, Z_{m}\right] \subset R$. Note that $R_{m}$ is a localization of $T$ and so $R_{m}=T_{\left(P_{m} \cap T\right)}$, and also note that $P_{m} \cap R \varsubsetneqq P$ implies $R_{P} \varsubsetneqq R_{m}$. Putting these facts together, we obtain $T_{P \cap T} \subset R_{P} \varsubsetneqq R_{m}=T_{\left(P_{m} \cap T\right)}$ and so $P_{m} \cap$ $T \varsubsetneqq P \cap T$. This yields an element $g \in P \cap T-P_{m} \cap T$. To continue the proof, we require $g$ to satisfy certain additional properties which this particular element may not. However, whenever $g^{\prime} \in P_{m} \cap T,\left(g-g^{\prime}\right) \in P \cap T-$ $P_{m} \cap T$ and so we may safely replace $g$ by $\left(g-g^{\prime}\right)$. We proceed thus. First note that $g$ is a sum of monomials in $T$ and those monomials containing $Y_{m}$ are in $P_{m}$. Delete them, leaving $g \in K\left[X, Y-\left\{Y_{m}\right\}, Z_{m}\right]$. Next express $g$ as a polynomial in $Z_{m}$ with coefficients in $K\left[X, Y-\left\{Y_{m}\right\}\right]$. As before, if any coefficient is in $P_{m}$, delete the appropriate term.

If $Z_{m} \notin P$, we may assume (dividing if necessary) that $g$ has nonzero constant term. Thus $g=a_{0} Z_{m}^{n}+\cdots+a_{n}$ with $v_{m}\left(a_{n}\right)=0$. Set $h=Y_{m}^{n} g=$ $a_{n} Y_{m}^{n}+\cdots+a_{0} f_{m}^{2 n}$ and observe the conditions we have forced upon $g$ are precisely those needed to conclude $Y_{m}^{n+1}+h \in S^{\prime}$. Thus $Y_{m}+g=$ $\left(1 / Y_{m}^{n}\right)\left(Y_{m}^{n+1}+h\right)$ is a unit of $R_{0}$. But it is also a unit of $R_{m}$ and so cannot be in $P$, contradicting $Y_{m}, g \in P$.

On the other hand, if $Z_{m} \in P$, then $Y_{m}+Z_{m}^{2} \in P$. However, $Y_{m}+Z_{m}^{2}=$ $\left(1 / Y_{m}^{2}\right)\left(Y_{m}^{3}+f_{m}^{4}\right)$ is a unit in both $R_{0}$ and $R_{m}$. This contradiction completes the proof of the claim and hence the lemma.

Now, according to a theorem of Heinzer and Ohm [2, Corollary 1.8, p. 295], whenever $R$ is the intersection of a family $\left\{R_{\alpha}\right\}$ of Noetherian flat $R$-algebras such that each ideal $I \subset R$ satisfies $I R_{\alpha} \neq R_{\alpha}$ for at least one and at most finitely many $R_{\alpha}$, then $R$ must be Noetherian. The hypothesis of this theorem has been verified by Lemmas 2,3 and 4 . Hence

\section{THEOREM 5. $R$ is Noetherian.}

LEMMA 6. If $u$ is a unit of $R_{0}$, then $v_{k}(u)$ is even for every $k>0$.

Proof. $R_{0}$ is a localization of $K[X, Y]$ at the nonzero elements of $(K[Y]) S$. Consequently, it is enough to check the lemma for elements of $K[Y]$ and for factors of elements in $S^{\prime}$. We claim that for each $k, P_{k} \cap K[X, Y]=\left(f_{k}, Y_{k}\right)$ $\subset\left(X, Y_{k}\right)$. To see this, first note $P_{k} \cap K\left[X, Y-\left\{Y_{k}\right\}\right]=\left(f_{k}\right)$. Also $Y_{k} \in P_{k}$ as before. Finally, $\left(f_{k}, Y_{k}\right)$ is the unique prime of $K[X, Y]$ containing $Y_{k}$ which lies over $f_{k} K\left[X, Y-\left\{Y_{k}\right\}\right]$.

If $u \in K[Y], u=Y_{k}^{m} f$ where $f$ is not divisible by $Y_{k}$. Clearly $f \notin\left(X, Y_{k}\right)$ and so $v_{k}(u)=2 m$.

If $s \in S^{\prime}$ and $k \neq m$ (used in defining $\left.s\right), s \notin\left(X, Y_{k}\right)$ and so $v_{k}(s)=0$. Thus, if $u$ is a factor of $s, v_{k}(u)=0$ as well. It only remains to consider $v_{m}(u)$.

Suppose $s=Y_{m}^{n+1}+\sum_{i=0}^{n} a_{i} Y_{m}^{i}$ and $s=u w$. Note $v_{m}(s)=2 n$. We may assume, factoring as polynomials in $Y_{m}$, that $u=Y_{m}^{d}+b_{d-1} Y_{m}^{d-1}+\ldots$ and $w=Y_{m}^{e}+c_{e-1} Y_{m}^{e-1}+\ldots$ Then $d+e=n+1$ and $a_{n}=b_{d-1}+c_{e-1}$. As $v_{m}\left(a_{n}\right)=0$, we may conclude that one of its summands has valuation zero, say $v_{m}\left(b_{d-1}\right)=0$. Now $v_{m}(u)$ is in fact $\min \left\{v_{m}\left(b_{i} Y_{m}^{i}\right)\right\}$. This can be seen by 
noting $v_{m}(u)=v_{m}\left(Z_{m}^{d} u\right)$. Then $Z_{m}^{d} u$ is a polynomial in $Z_{m}$ with coefficients in $K\left[X, Y-\left\{Y_{m}\right\}\right]$ and $f_{m}$ divides such a polynomial if and only if it divides each coefficient. Thus $v_{m}\left(Z_{m}^{d} u\right)$ will be the minimum of the valuations of the coefficients, which are $\left\{b_{i} f_{m}^{2 i}\right\}$ and $v_{m}(u)$ will be as claimed. Then $v_{m}(u) \leqslant$ $v_{m}\left(b_{d-1} Y_{m}^{d-1}\right)=2(d-1)$ and $v_{m}(w) \leqslant v_{m}\left(Y_{m}^{e}\right)=2 e$. As $v_{m}(u)+v_{m}(w)=2 n$ $=2(d-1)+2 e$, we see that both $u$ and $w$ have even valuations.

Finally, since $f_{m}^{4}$ is not a cube, $s=Y_{m}^{3}+f_{m}^{4}$ is irreducible. So we need only consider $v_{m}(s)=4$. This completes the proof.

THEOREM 7. The ideal $\left(X_{1}, X_{2}\right) R$ is a height two prime and so has a regular system of parameters. However, it contains no principal height one primes and so does not have a prime sequence.

Proof. To prove $I=\left(X_{1}, X_{2}\right) R$ is a height two prime, we need only show $I=\left(X_{1}, X_{2}\right) R_{0} \cap R$. By [2, 1.7], it suffices to show $I R_{k}=\left(\left(X_{1}, X_{2}\right) R_{0} \cap\right.$ $R) R_{k}$ for each $k$. However, for $k>0, I R_{k}=R_{k}=\left(\left(X_{1}, X_{2}\right) R_{0} \cap R\right) R_{k}$. Also $I R_{0}=\left(X_{1}, X_{2}\right) R_{0}=\left(\left(X_{1}, X_{2}\right) R_{0} \cap R\right) R_{0}$. So the first sentence has been shown.

Let $Q$ be any height one prime contained in $I$. Then $Q=f_{j} R_{0} \cap R$ for some $f_{j}$. If $Q=h R$, then $h R_{0}=Q R_{0}=f_{j} R_{0}$ and so $h=f_{j} u$ where $u$ is a unit in $R_{0}$. However, $v_{j}(h)=v_{j}\left(f_{j}\right)+v_{j}(u)=1+v_{j}(u)$ is odd and consequently nonzero. Since $h \in R, v_{j}(h) \geqslant 0$ and so $v_{j}(h)>0$, i.e., $h \in P_{j} \cap R$. But $Q \notin P_{j} \cap R$ and so $Q$ cannot be principal.

\section{REFERENCES}

1. E. Davis, Prime ideals and prime sequences in polynomial rings, Proc. Amer. Math. Soc. 72 (1978), 33-38.

2. W. Heinzer and J. Ohm, Noetherian intersections of integral domains, Trans. Amer. Math. Soc. 167 (1972), 291-308.

3. R. Heitmann, Prime ideal posets in Noetherian rings, Rocky Mountain J. Math. 7 (1977), $667-673$.

Department of Mathematics, University of TeXas, Austin, TeXas 78712 